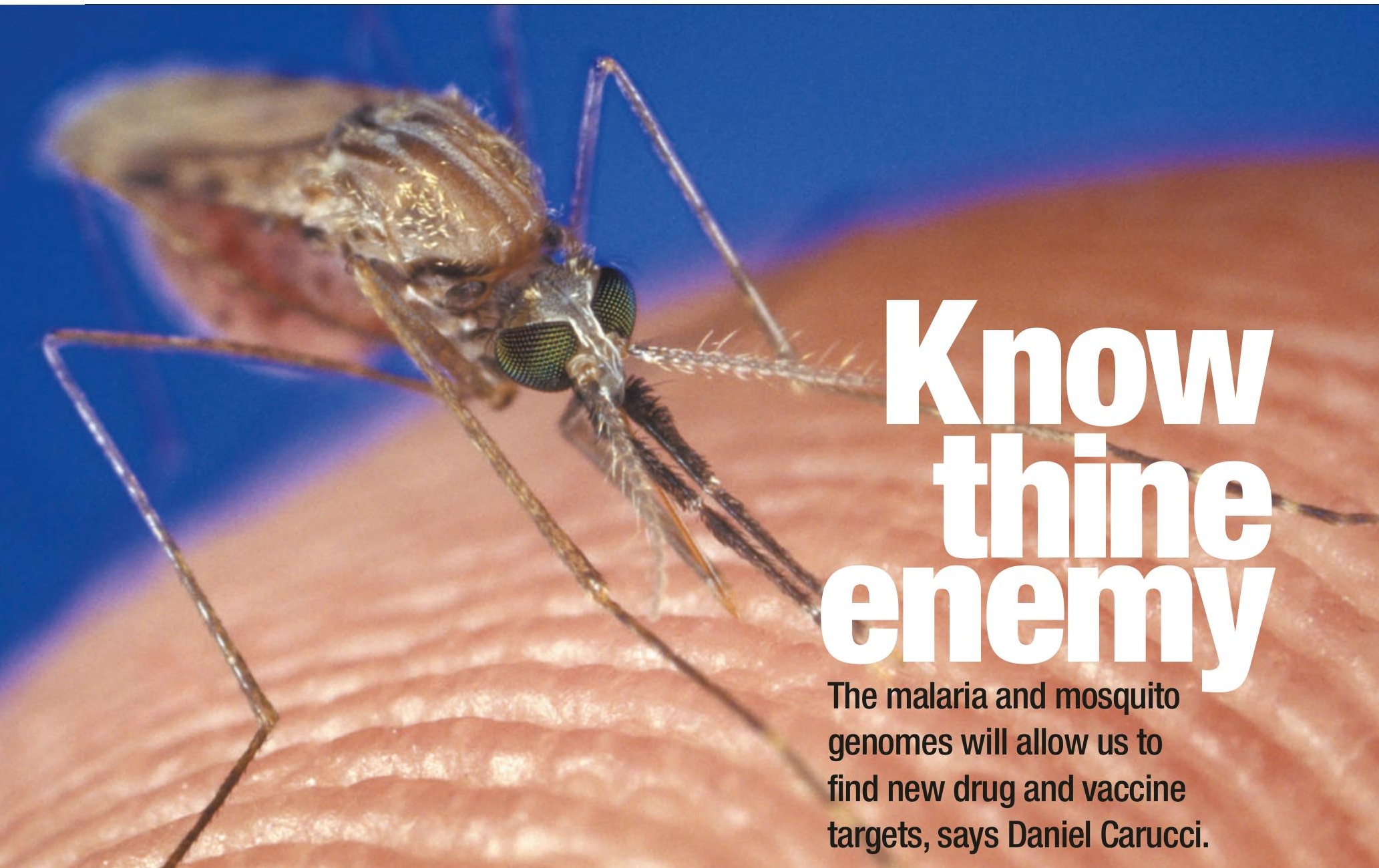

he deciphering of the complete genome of the malaria parasite ushers in entirely new approaches to developing vaccines and drugs against this ancient pathogen. To conquer, or even control, malaria we need to fully understand the parasite's biology. Co-evolving with us over millennia, it has developed sophisticated tactics to survive our immune defences, and more than any other disease has spurred changes to the human genome itself.

The deadliest of the malaria parasites, Plasmodium falciparum, is thought to have originated more than 100,000 years ago, coinciding with the geographical expansion of humans out of Africa. We have been battling it ever since.

The parasite infects red blood cells, feeds on haemoglobin and eventually destroys the cells, often resulting in severe anaemia. It also makes the cells' surfaces sticky so that they cling to the lining of blood vessels, clump together and clog narrow arteries, blocking blood to vital organs including the brain. In the latter, 'cerebral malaria' can lead to coma and death.

The threat posed by malaria has been so great that, at the risk of potentially fatal sickle-cell anaemia in homozygous individuals, humans have evolved a mutant form of the haemoglobin gene that produces an abnormally folded protein the parasite cannot digest. The fact that this deleterious mutation persists in our genome is a sign of the enormous pressure that malaria exerts over our evolution.

The continued success of the parasite - it infects more people today than ever before is due in part to an ability to alter its surface proteins to deceive the host immune system, and to suppress immune responses to its quiescent liver stage (see 'Save the children', page 940). To develop vaccines and drugs that exploit vulnerabilities in the parasite's biology necessitates a complete understanding of the parasite and its complex relationships with its human and mosquito hosts.

\section{Cracking the code}

The genetic code of $P$. falciparum, published in 2002 (ref. 1), has yet to spawn extraordinary breakthroughs, but it has energized the malaria community and has also attracted a much broader range of scientists to join the effort. They have brought technologies such as gene chips, proteomics and comparative genomics. We now have the foundations necessary to study global gene and protein expression in the parasite, identify genetic polymorphisms, uncover mechanisms of drug resistance and elucidate the causes of severe disease.
The best way to capitalize on the malaria genome will be through partnerships and consortia that bring together bioinformaticians, biologists, vaccine and drug developers, and physicians working in endemic areas. These could provide a set of 'credentials' for each malaria gene, indicating, for example, what controls its temporal expression, variation between related species in populations and role in the parasite life cycle. This would allow researchers to prioritize targeted approaches to drug and vaccine development.

Gene chips have already been used to determine how each of the more than 5,400 parasite genes is turned on and off in the redblood-cell stage of the life cycle ${ }^{2,3}$, and to assign functions to particular proteins ${ }^{2}$. We must discover how these genes evolved to counter human defences, and in particular those genes that the parasite is forced to alter to avoid the immune system and which represent potential vaccine targets.

The same gene-chip technologies that have been used to study genetic diversity in model systems such as yeast are helping to identify mutations in malaria parasite strains that may be associated with parasite virulence or those under immune selection ${ }^{4}$. Gene chips also have the potential to reveal how the parasite responds to drugs and to 
identify mutants that confer resistance. This could help elucidate mechanisms of drug resistance and, ultimately, ways to combat it. So far, with only chloroquine tested, few changes have been identified at the RNA transcript level in response to this one drug. But changes in gene expression are likely to be seen with other drugs.

\section{Pattern analysis}

Proteomics - the study of the entire range of proteins produced by genes - is an exciting new field, and researchers are applying technologies developed in model organisms such as yeast to each stage in the life cycle of Plasmodium ${ }^{5,6}$. Using microliquid chromatography and highly sensitive mass spectrometry, for example, scientists can catalogue the identity and location of the thousands of proteins present at each stage, including proteins expressed on the surface of infected red blood cells.

Patterns of protein expression can identify antigens that would make suitable targets for vaccines. For example, proteomics has been used to identify vaccine candidates in the sporozoite stage, by pinpointing the antigens that generate cellular immune responses in volunteers protected against infection by an attenuated sporozoite vaccine $e^{7,8}$.

Plasmodium is one of the 'apicomplexan' parasites, which possess a vestigial chloroplast. But unlike a related parasite in this

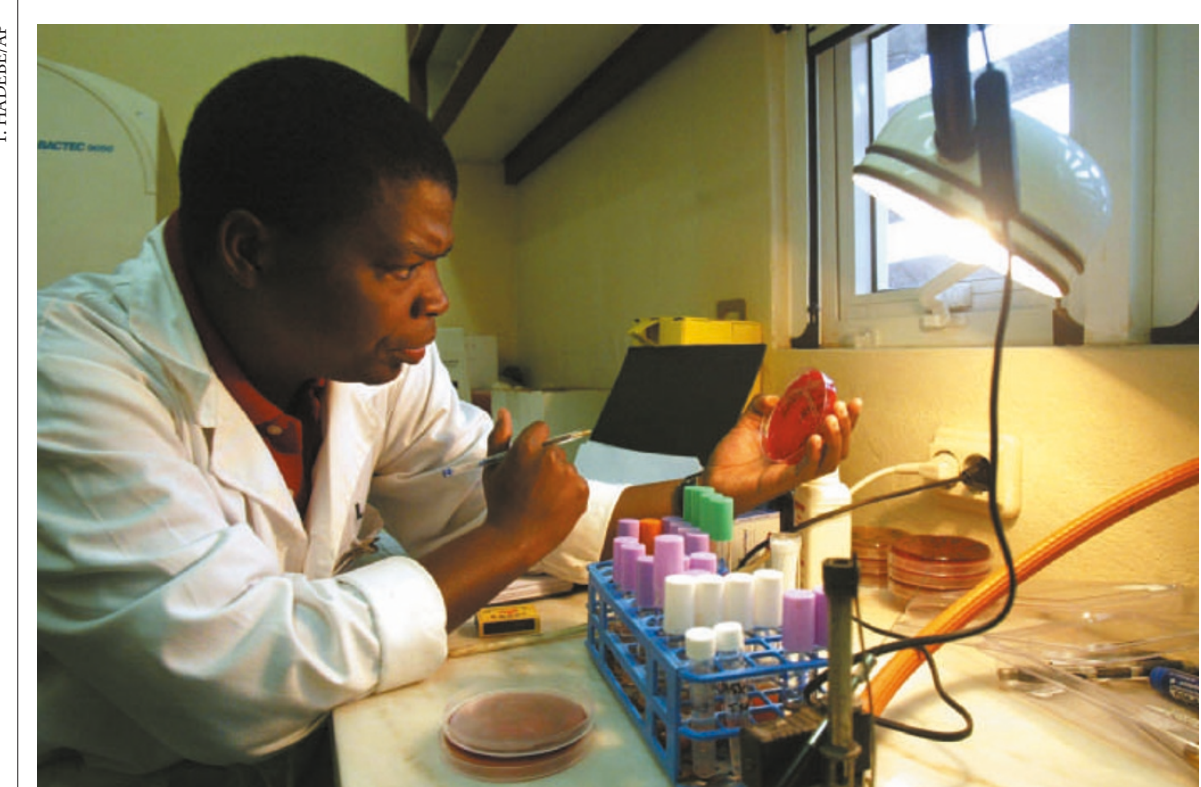

\section{"Our malaria adversary \\ "Our malaria adversary
has evolved an impressive array of defences. Genomics will be critical to breaching them."} which can infect all eukaryotic cell types, Plasmodium is remarkable in that its different

families are specific to particular vertebrate hosts, such as humans, mice, birds or lizards.

Understanding this specificity is key. New intervention strategies might be developed based on the mechanisms underlying this preference for different species and host cells. Why are human red blood cells susceptible to $P$. falciparum but not to related rodent malaria species? How do parasites recognize host and target cells?

Proteins that are differentially expressed

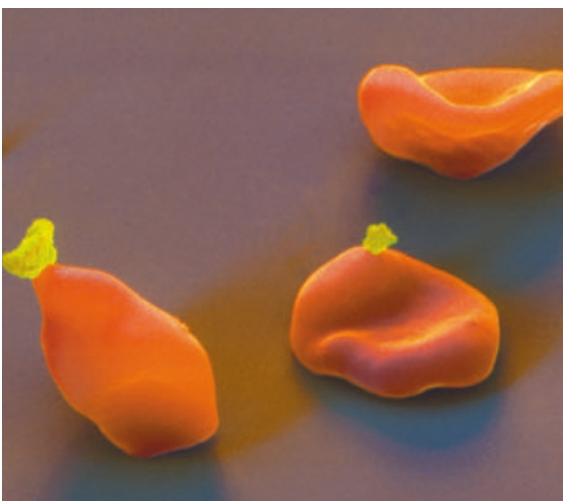

Plasmodium falciparum (yellow) emerges from red bloods that it has infected (above), while a researcher in Mozambique conducts research into how to combat the parasite. on the surface of P. falciparum sporozoites and merozoites, for example, enable invasion of liver cells or red blood cells, respectively. But despite much research in the pre-genomics era, only a handful of molecules potentially involved in invasion are known, including those in the reticulocytebinding homologue family ${ }^{9}$ that allow merozoites spewing from the liver to recognize red blood cells.

Comparative genomics may shed light on parasite-host interactions ${ }^{10}$ and lead to entirely new ways to interrupt crucial steps in the parasite life-cycle, perhaps using small molecules. Combining comparative genomics with gene-expression studies may identify new targets for drugs lethal to the parasite but non-toxic to humans.

In addition, it may be possible to extend the useful life of drugs by pinpointing drug resistance mechanisms, making the high costs of drug development a better investment. Although a daunting challenge, it may also be possible to investigate at the genomic level, in combination with case-control field trials, the role of certain malaria parasites in causing severe anaemia and fatal neurological complications.

Within the next five years, the sequences of the remaining five species of parasite will be completed, as well as those of several more mosquito vectors. This should help to identify the key molecules making the parasite specific to its host, and possible Achilles' heels.

But doing molecular genetics in the parasite is challenging because of the difficulties in culturing its various stages, and large-scale gene knockout experiments are fraught with formidable technical challenges. Our malaria adversary has evolved an impressive array of defences. Genomics will be critical to breaching them, but it's going to be a long and hard campaign.

Daniel Carucci is director of Grand Challenges in Global Health at the Foundation for the National Institutes of Health, Bethesda, Maryland.

1. Gardner, M. J. et al. Nature 419, 498-511 (2002).

2. Le Roch, K. G. et al. Science 301, 1503-1508 (2003).

3. Bozdech, Z. et al. PLoS Biol. 1, E5 (2003).

4. Volkman, S. K. et al. Science 298, 216-218 (2002).

5. Florens, L. et al. Nature 419, 520-526 (2002).

6. Lasonder, E. et al. Nature 419, 537-542 (2002).

7. Doolan, D. L. et al. J. Exp. Biol. 206, 3789-3802 (2003).

8. Doolan, D. L. et al. Proc. Natl Acad. Sci. USA 100, 9952-9957 (2003).

9. Duraisingh, M. T. et al. EMBO J. 22, 1047-1057 (2003). 10. Cowman, A. F. \& Crabb, B. S. Science 298, 126-128 (2002) 